\title{
Diffusion tensor imaging (tractography) in elderly people with dementia type Alzheimer's disease and mixed dementia
}

\author{
Paulina E. Bombón-Albán ${ }^{1}$, Alberto J. Mimenza-Alvarado², Oscar R. Marrufo-Meléndez³, \\ Johnatan Rubalcava-Ortega ${ }^{4}$, Lidia A. Gutiérrez-Gutiérrez ${ }^{5}$, and Sara G. Aguilar-Navarro ${ }^{6}$ \\ ${ }^{1}$ Departament of Neurology, Neuromedicenter, Quito, Ecuador; ${ }^{2}$ Departament of Geriatrics, Instituto Nacional de Ciencias Médicas y Nutrición \\ Salvador Zubirán (INCMyNSZ), Mexico City; ${ }^{3}$ Departament of Neuroimaging, Instituto Nacional de Neurología y Neurocirugía Manuel Velasco \\ Suárez, Mexico City; ${ }^{4}$ Departament of Neuroimaging, INCMyNSZ, Mexico City; ${ }^{5}$ Departament of Neurology, INCMyNSZ, Mexico City; ${ }^{6}$ Departament \\ of Geriatrics, INCMyNSZ, Mexico City. Mexico
}

\begin{abstract}
Objective: The objective of the study was to evaluate the characteristics of white matter tracts by diffusion tensor imaging (tractography) in elderly people with dementia type Alzheimer's disease (AD) and mixed dementia through the measurements of fractional anisotropy (FA) and mean diffusivity (MD). Methods: Eight patients with $A D$ and eight patients with mixed dementia were studied. Clinical, cognitive, and neuroimaging evaluation was carried out. Variables are described using the arithmetic mean, standard deviations, and frequencies. Chi-square and U Mann-Whitney tests were used. Correlation analysis was performed between neuropsychological characteristics and the degree of affection by tractography (FA and MD). Results: Significant differences were found between the groups of $A D$ versus mixed dementia in FA: right cerebral peduncle 0.5733 versus 0.5557 ( $p<0.05)$, left cerebral peduncle 0.5744 versus $0.5476(p<0.01)$, right external capsule 0.3619 versus $0.3346(p<0.01)$, and left cingulum gyrus 0.4049 versus $0.3756(p<0.05)$. MD: right thalamic posterior radiation 0.0016 versus $0.0010(p<0.03)$ and left external capsule 0.0015 versus $0.0012(p<0.03)$. Conclusion: Using tractography, it is possible to quantify the extent of damage to the white matter tracts (vascular and neurodegenerative).
\end{abstract}

Key words: Tractography. Fractional anisotropy. Mean diffusivity. Alzheimer's disease. Mixed dementia.

\section{Imagen de tensor de difusión (tractografía) en personas mayores con demencia tipo enfermedad de Alzheimer y demencia mixta}

\section{Resumen}

Objetivo: Evaluar las características de los tractos de sustancia blanca por imagen de tensor de difusión (tractografía) en personas mayores con demencia tipo enfermedad de Alzheimer y demencia mixta, a través de la medición de la fracción de anisotropía y la difusividad media. Métodos: Se estudiaron 8 pacientes con demencia tipo enfermedad de Alzheimer y 8 pacientes con demencia mixta, se realizó evaluación clínica, cognitiva y neuroimagen. Las variables se describen utilizando media aritmética, desviaciones estándar, Chi-cuadrada y U Mann-Whitney. Se realizó análisis de correlación entre características neuropsicológicas y el grado de afección por tractografía (fracción de anisotropía y difusividad media). Resultados: Se encontraron diferencias significativas entre los grupos enfermedad de Alzheimer vs. demencia mixta en la fracción de anisotropía: pedúnculo cerebral derecho 0.5733 vs. 0.5557 ( $p<0.05)$, pedúnculo cerebral izquierdo 0.5744 vs.

\section{Correspondence:}

*Sara G. Aguilar Navarro

E-mail: sgan30@ hotmail.com
Available online: 17-03-2021

Rev Mex Neuroci. 2021;22(2):42-50 www.revmexneurociencia.com 2604-6180/ (c) 2020 Academia Mexicana de Neurología A.C. Published by Permanyer. This is an open access article under the CC BY-NC-ND license (http://creativecommons.org/licenses/by-nc-nd/4.0/). 
$0.5476(p<0.01)$, cápsula externa derecha 0.3619 vs $0.3346(p<0.01)$ y giro del cíngulo izquierdo 0.4049 vs 0.3756 $(p<0.05)$. Difusividad media: radiación posterior talámica derecha 0.0016 vs. $0.0010(p<0.03)$ y cápsula externa izquierda 0.0015 vs. 0.0012 ( $p$ < 0.03). Conclusión: Mediante la tractografía es posible cuantificar la magnitud de daño de los tractos de la sustancia blanca (vascular y neurodegenerativo).

Palabras clave: Tractografía. Fracción de anisotropía. Difusividad media. Enfermedad de Alzheimer. Demencia mixta.

\section{Introduction}

Tractography is used to correlate the integrity of white matter tracts with cognitive function in cognitively healthy people and with dementia ${ }^{1}$. It is a special technique of simple magnetic resonance imaging (MRI), which consists of non-invasive diffusion tensor imaging (DTI), sensitive to the diffusion of water molecules ${ }^{2}$, which allows a live three-dimensional reconstruction of the tracts within the central nervous system ${ }^{1,3}$. The fibers of white matter are classified into three categories: (a) association fibers: those that interconnect cortical areas of the same hemisphere, (b) commissural fibers: interconnect areas between both hemispheres, and (c) projection fibers, which connect the cortex with formations of lower levels 4 .

The interruption of the tracts can be detected as a decrease in fractional anisotropy (FA) (diffusion orientation and directionality) and an increase in mean diffusivity (MD) (degree of water diffusion) $)^{1,4,5,6}$. An anisotropy of "0" corresponds to a perfect sphere, while 1 would be an ideal linear diffusion. Well-defined tracts generally have an FA greater than $0.2^{1,6,7}$. Preliminary studies show a decrease in white-matter connections in patients with Mild cognitive impairment and Alzheimer's disease (AD). Especially noticeable is the increased MD and decreased FA in these patients compared to normal controls. These changes were found in the posterior cingulate fasciculus, the uncinate fasciculus, or both at once ${ }^{1}$.

$A D$ is the most common neurodegenerative disorder that causes dementia in elderly individuals. The cause of $A D$ is unknown in most cases. The most powerful risk factor for developing $A D$ is age, with $A D$ affecting as many as $40-50 \%$ of individuals older than 85 years $^{8}$. Mixed dementia (MixD) represents the third leading cause of dementia, only behind $A D$ and vascular dementia $(\mathrm{VaD})$. The prevalence range of MixD varies between $20 \%$ and $40 \% \%^{9,10}$. The Vascular Impairment of Cognition Classification Consensus Study (VICCCS) proposes in its classification of vascular cognitive impairment $(\mathrm{VCl})$ the definition of MixD, which includes phenotypes that represent the combination of vascular and neurodegenerative diseases, that is, VCl-AD, in addition to other possible combinations ${ }^{11}$. From a clinical viewpoint, loss of memory (especially episodic and semantic) is considered a typical feature of $A D$, whereas executive dysfunction has traditionally been associated with MixD. Several MRI analysis methods can track structural atrophy in $A D$ and the presence of ischemic lesions on computed tomography or MRI is key in the diagnosis of individuals with MixD9 .

Populations with high cardiovascular risk, patterns are more frequently observed cognitive skills of mixed type and the pure forms of dementia or sole are not the first diagnostic option in older subjects with memory complaints. This aspect is important because the adequate identification of these potentially risk factors modifiable could help intervene in an entity still without a definitive therapeutic route ${ }^{9}$. Accurate diagnosis of $A D$ and MixD is of crucial significance for epidemiological purposes and for preventive and therapeutic strategies $^{12}$.

The objective of our study was to evaluate the characteristics of white matter tracts by DTI (tractography) in elderly people with dementia type $A D$ and MixD through the measurements of FA and MD.

\section{Methods}

\section{Type of study and patients}

Cross-sectional study carried out in a memory disorder clinic of a third-level hospital in Mexico City. All the patients were recruited in a period from March 2019 to December 2019. They all signed a consent form and underwent clinical and cognitive evaluations by a specialist in neurology and/or geriatrics. From the comprehensive geriatric evaluation, sociodemographic variables were obtained such as sex, age, education, Katz In$\operatorname{dex}^{13}$, Lawton and Brody Scale ${ }^{14}$, and Yesavage Geriatric Depression Scale (GDS) ${ }^{15}$. The neuropsychological evaluation consisted of applying the NEUROPSI test ${ }^{16}$, Mini-Mental State Examination (MMSE) ${ }^{17}$, verbal fluency test $^{18}$, clock-drawing test ${ }^{19}$, frontal assessment battery $(F A B)^{20}$, and the Clinical Dementia Rating (CDR) Scale ${ }^{21}$. 
Patients with visual and auditory acuity deficits that would make it impossible to apply neuropsychological tests were excluded, as well as patients with dysthyroidism (hyper or hypothyroidism) without treatment, hypertension, dyslipidemia and/or uncontrolled diabetes mellitus, and those with glycosylated hemoglobin levels higher than $9 \%$, patients with severe hypoglycemia, other causes of dementia (vascular, frontotemporal, Lewy bodies), other uncontrolled medical conditions (cardiovascular, renal, or advanced lung disease), inflammatory diseases, active smokers, patients with significant depressive symptoms (GDS score > 5/15), CDR $>1$, and the presence of metallic objects, devices, or conditions that would make MRI impossible.

\section{Clinical diagnosis of dementia}

The patients were classified into two groups: $A D$ $\mathrm{n}=8$ and MixD $\mathrm{n}=8$, according to their performance in neuropsychological evaluation and current clinical criteria. For the diagnosis of dementia type $A D$, the criteria of the Diagnostic and Statistical Manual of Mental Disorders version 5 (American Psychiatric Association DSM-5) ${ }^{22}$ and the criteria of the National Institute of Neurological and Communicative Disorders and Stroke - Alzheimer's Disease and Related Disorders Association (NINCDS-ADRDA) ${ }^{23}$ were used. For the diagnosis of MixD, the VICCCS ${ }^{11}$ criteria were used. For the dementia stage, the CDR score (Mild $=1$ ) was applied in both conditions.

\section{Magnetic resonance DTI (tractography)}

All patients had a simple brain MRI, with $\mathrm{T} 1$ sequence with DTI and volumetry. The images were obtained with a $1.5 \mathrm{~T}$ resonator (Siemens ${ }^{\circledR}$ Medical Systems). Image processing was performed by the Neuroimaging Department of the National Institute of Medical Sciences and Nutrition, Salvador Zubirán (INCMNSZ). The MRI was evaluated by a neuroradiologist (not knowing of patients being in the clinical group). A high-resolution anatomical scanner was used with a T1-weighted three-dimensional magnetization sagittal sequence, prepared with fast gradient echo (MP-RAGE), field of view (FoV) $26 \mathrm{~mm}$, spatial resolution $1 \times 1 \times 1 \mathrm{~mm}^{3}$, repetition time of $1500 \mathrm{~ms}$ (TR), echo time (TE), rotation angle $9^{\circ}$, number of cuts 176, with 25 independent diffusion gradient directions ${ }^{1,4,5,24}$. The process to perform the tractography begins with the definition of an origin point (also called seed point), and the reconstruction process, which consists of taking the orientation of the voxel and advancing a certain distance until a new direction of propagation is found with the help of the orientation of neighboring voxels. From here on, it continues iteratively, adding a new segment to each step, thus forming a path with the points in each step ${ }^{3,25}$. The basis of the anisotropic diffusion of water in the nervous system is due to the involvement of longitudinally oriented structures within the axons, such as myelin, axonal membrane, microtubules, neurofilaments, or axonal transport, which hinders the diffusion of water. MD measures the extent of diffusion in a voxel; the increase in MD may be related to a loss of coherence in fiber alignment, a lower fiber density, or a loss of myelination ${ }^{1,6,8}$.

The images obtained from DTI were assigned to maps of FA and MD, the tracts were established using JHU White Matter Tractography Atlas. A standard color scheme was used in the software to encode the FA maps, with blue indicating superior-inferior, red indicating transverse, and green indicating anterior-posterior ${ }^{26}$. Tracts were created in the Diffusion Toolkit using the Continuous Tracking Fiber Assignment method with $35^{\circ}$ angle threshold and a unique set of fibers was generated for each patient by performing anatomically detailed analysis. It was decided to study tracts that correspond to association, commissural and projection fibers (16 brains, 48 regions of interest $-\mathrm{ROI}$ ). Mean values of $F A$ and $M D$ were analyzed for each tract, these being the most important indices for determining dementia according to the previous studies ${ }^{1,6}$. In Figure 1, an example of the tract obtained with the proposed protocol is shown, where the cingulum is seen in red, corresponding to the left cerebral hemisphere.

\section{Structural MRI}

\section{Cortical thickness studies}

The T1 images were processed with the FreeSurfer 5.3 program, which is capable of constructing models of the cortical surface of the nervous system. This software works in an automated way and the process consists of registering the input volumes determined by the user to the atlas MNI305 (Montreal National Institute), then it performs an intensity normalization and a segmentation of the voxels according to this parameter and their spatial location to classify them in different types of tissue: gray matter, white matter, and cerebrospinal fluid ${ }^{27}$. For the present study, cerebral cortex was divided into 48 ROls based on turns per hemisphere, and in this manner, the geometric information derived from 


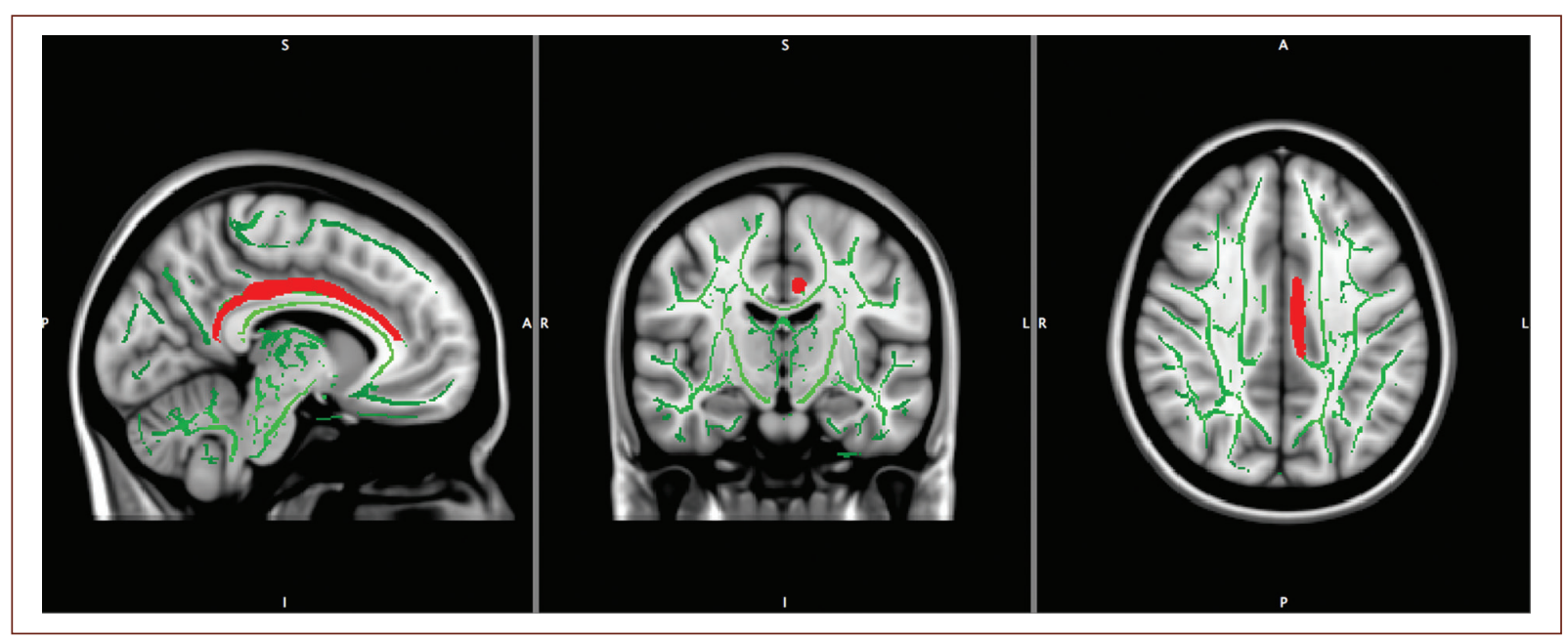

Figure 1. Left cingulum tractography (red).

the cortical model with the labels of the neuroanatomical parceling is incorporated.

The entire protocol was acquired during a single 25-min session, where the patient's brain was aligned in the stereotactic space: the anterior commissure-posterior commissure line was aligned with the axial plane, and the interhemispheric fissure was aligned along the sagittal plane and at right angles to the coronal plane.

\section{Statistics analysis}

The variables are described by using arithmetic mean and standard deviations. The Chi-square test was used for categorical variables and the Mann-Whitney $U$ test was used for continuous variables. Clinical and demographic data were tested for normality using a Shapiro-Wilk test. The voxel statistics in the skeletonized images were performed using the randomized, an FSL tool for nonparametric permutation inference in neuroimaging data. The carcass of mean FA (threshold 0.2 ) was used as a mask, the permutation number was set at 5000, meaning the difference between groups was corrected for multiple comparisons using the no-threshold cluster enhancement method (TFCE) and tested a $p<0.01$. To identify the power of the selected brain areas to discriminate between $A D$ and MixD, a stepwise discriminant analysis was performed using the dementia subgroup (AD or MixD) as the dependent variable and the mean values of ROI of $F A$ and $M D$ as independent variables. Finally, by means of a Spearman correlation, the differences between the characteristics of the microstructure of the brain regions were searched in FA, MD, and the neuropsychological evaluation. Variables with a value of $p<0.05$ were taken as significant.

SPSS version 22 for Windows ${ }^{\circledR}$ (SPSS Inc., Chicago, Illinois) was used for the analyses. The protocol was approved by the institutional Ethics Committee (REF. 3009).

\section{Results}

The average age of the AD group members was 84.3 \pm 8.8 years old and of the MixD group, it was $85.3 \pm$ 7.6 years old $(p<0.57)$ and $69 \%$ were women $(p<0.23)$. The average schooling of $A D$ patients was $10.2 \pm 7.3$ and of MixD was $11.3 \pm 4.8(p<0.87)$. In the AD group, $38 \%$ were hypertensive versus $100 \%$ in $\operatorname{MixD}(p<0.00)$ and none had obesity in the AD group versus $38 \%$ in MixD $(p<0.05)$. Regarding the global neuropsychological assessment, patients with AD had a worse cognitive performance in the cognitive assessment (NEUROPSI) $68.5 \pm 11.6$ points compared to the MixD group $83.2 \pm 12.4(p<0.02)($ Table 1$)$.

\section{DTI (Tractography)}

Regarding the tractography findings, significant differences were found in $F A$ between the $A D$ versus MixD groups in the following tracts: right cerebral peduncle 0.5733 versus $0.5557(p<0.05)$, left cerebral peduncle 0.5744 versus $0.5476(p<0.01)$, right external capsule 0.3619 versus $0.3346(p<0.01)$, and left cingulum gyrus 0.4049 versus $0.3756(p<0.05)$. Regarding $M D$, the differences found were in the following tracts: right thalamic posterior radiation 0.0016 versus 
Table 1. Sociodemographic characteristics and cognitive performance in Alzheimer's disease and mixed dementia

\begin{tabular}{|c|c|c|c|c|}
\hline & Total patients $(n=16$ ) & Alzheimer's disease $(n=8)$ & Mixed dementia ( $\mathrm{n}=8$ ) & $\mathbf{p}$ \\
\hline Age years & $84.8 \pm 7.9$ & $84.3 \pm 8.8$ & $85.3 \pm 7.6$ & 0.57 \\
\hline Sex (Female) \% & (11) 69 & (4) 25 & (7) 44 & 0.23 \\
\hline Years of education, $\%$ & $10.8 \pm 6.0$ & $10.2 \pm 7.3$ & $11.3 \pm 4.8$ & 0.87 \\
\hline Hypertension \% & (11) 69 & (3) 38 & (8) 100 & 0.00 \\
\hline Mellitus diabetes $\%$ & (3) 19 & (1) 13 & (2) $25 \%$ & 0.52 \\
\hline Heart disease \% & (4) 25 & (1) 13 & (3) 38 & 0.24 \\
\hline Cerebral vascular event \% & (1) 6 & (0) 0 & (1) 13 & 0.30 \\
\hline Dyslipidemia \% & (9) 56 & (3) 38 & (6) 75 & 0.13 \\
\hline Hypothyroidism \% & (4) 25 & (1) 13 & (3) 38 & 0.24 \\
\hline Obesity \% & (3) 19 & (0) 0 & (3) 38 & 0.05 \\
\hline History depression \% & (4) 25 & (2) 25 & (2) 25 & 1.00 \\
\hline GDS - 15 & $2.0 \pm 1.5$ & $2.6 \pm 1.5$ & $2.2 \pm 1.6$ & 0.72 \\
\hline $\begin{array}{l}\text { Functionality } \\
\text { KATZ } \\
\text { LB }\end{array}$ & $\begin{array}{l}6.0 \pm 0.2 \\
6.5 \pm 2.5\end{array}$ & $\begin{array}{c}6 \pm 0.0 \\
5.3 \pm 2.9\end{array}$ & $\begin{array}{l}5.8 \pm 0.3 \\
5.2 \pm 2.3\end{array}$ & $\begin{array}{l}0.72 \\
0.72\end{array}$ \\
\hline NEUROPSI total & 75. $8 \pm 13.9$ & $68.5 \pm 11.6$ & $83.2 \pm 12.4$ & 0.02 \\
\hline Orientation & $4.0 \pm 1.7$ & $5.1 \pm 1.3$ & $2.8 \pm 1.4$ & 0.02 \\
\hline Attention & $13.8 \pm 5.2$ & $13.88 \pm 4.9$ & $13.75 \pm 5.8$ & 0.72 \\
\hline Memory & $20.6 \pm 5.4$ & $23.3 \pm 4.9$ & $17.8 \pm 4.7$ & 0.03 \\
\hline Language & $23.1 \pm 4.1$ & $21.7 \pm 5.1$ & $24.5 \pm 2.6$ & 0.23 \\
\hline Visuospatiality/visuospatiality & $9.22 \pm 1.5$ & $9.1 \pm 1.6$ & $9.3 \pm 1.6$ & 0.87 \\
\hline Executive functions & $11.5 \pm 3.5$ & $11.8 \pm 3.6$ & $11.2 \pm 3.6$ & 0.50 \\
\hline MMSE & $22.5 \pm 3.2$ & $22.6 \pm 3.2$ & $22.5 \pm 3.5$ & 0.03 \\
\hline Semantic verbal fluency (animals) & $11.0 \pm 3.8$ & $13.0 \pm 2.8$ & $9.1 \pm 3.8$ & 0.06 \\
\hline Phonological verbal fluency (letter F) & $8.9 \pm 3.8$ & $10.6 \pm 4.0$ & $7.0 \pm 3.5$ & 0.05 \\
\hline Clock-drawing test & $2.3 \pm 1.7$ & $2.5 \pm 2.0$ & $2.13 \pm 1.3$ & 0.95 \\
\hline FAB & $12.4 \pm 3.2$ & $12.88 \pm 3.9$ & $12.0 \pm 2.5$ & 0.44 \\
\hline
\end{tabular}

GDS - 15: Yesavage Geriatric Depression Scale; LB: Lawton and Brody scale; MMSE: Mini-Mental State Examination; FAB: front evaluation battery.

$0.0010(p<0.03)$ and capsule left external capsule 0.0015 versus $0.0012(p<0.03)$ (Table 2).

\section{Correlations between cognitive domains and DTI parameters}

A correlation was observed between the cognitive domains and the tractography parameters (FA and $M D)$ in both groups, finding a moderate correlation in the $A D$ group between orientation and left cerebral peduncle with a Rho of $0.72(p<0.04)$; in the MixD group between orientation and left cingulate gyrus with a Rho of 0.73 $(p<0.04)$, memory and left cerebral peduncle with a Rho of $-0.70(p<0.05)$, and phonological fluency and left cerebral peduncle with a Rho of $-0.72(p<0.04)$ (Table 3$)$.

\section{Cortical thickness studies}

In the volumetric analysis, significant differences were found in the following ROls between $A D$ and MixD: Brain segmentation volume $964.92 \mathrm{~cm}^{3}$ versus $877.95 \mathrm{~cm}^{3}(p<0.02)$, brain segmentation volume 
Table 2. Comparison of tracts, fractional anisotropy, and mean diffusivity in Alzheimer's disease and mixed dementia

\begin{tabular}{|c|c|c|c|c|}
\hline & Total Patients $(n=16)$ & Alzheimer's disease $(n=8)$ & Mixed Dementia ( $\mathrm{n}=8$ ) & $\mathbf{p}$ \\
\hline $\begin{array}{l}\text { Fractional anisotropy } \\
\text { Right cerebral peduncle } \\
\text { Left cerebral peduncle } \\
\text { Right external capsule } \\
\text { Left cingulum gyrus }\end{array}$ & $\begin{array}{l}0.5645 \pm 0.0172 \\
0.5610 \pm 0.0215 \\
0.3483 \pm 0.0246 \\
0.3902 \pm 0.0271\end{array}$ & $\begin{array}{l}0.5733 \pm 0.0176 \\
0.5744 \pm 0.0209 \\
0.3619 \pm 0.0246 \\
0.4049 \pm 0.0224\end{array}$ & $\begin{array}{l}0.5557 \pm 0.0121 \\
0.5476 \pm 0.0121 \\
0.3346 \pm 0.0163 \\
0.3756 \pm 0.0241\end{array}$ & $\begin{array}{l}0.05 \\
0.01 \\
0.01 \\
0.05\end{array}$ \\
\hline $\begin{array}{l}\text { Mean diffusivity } \\
\text { Right thalamic posterior radiation } \\
\text { Left external capsule }\end{array}$ & $\begin{array}{l}0.0013 \pm 0.0004 \\
0.0013 \pm 0.0003\end{array}$ & $\begin{array}{l}0.0016 \pm 0.0005 \\
0.0015 \pm 0.0003\end{array}$ & $\begin{array}{l}0.0010 \pm 0.0000 \\
0.0012 \pm 0.0001\end{array}$ & $\begin{array}{l}0.03 \\
0.03\end{array}$ \\
\hline
\end{tabular}

Table 3. Spearman correlation between neuropsychological testing and DTI parameters in Alzheimer's disease and mixed dementia

\begin{tabular}{|c|c|c|c|c|c|c|c|c|c|c|c|c|}
\hline & \multicolumn{6}{|c|}{ Alzheimer's disease } & \multicolumn{6}{|c|}{ Mixed dementia } \\
\hline & \multicolumn{2}{|c|}{ Orientation } & \multicolumn{2}{|c|}{ Memory } & \multicolumn{2}{|c|}{$\begin{array}{c}\text { Fluency } \\
\text { phonological }\end{array}$} & \multicolumn{2}{|c|}{ Orientation } & \multicolumn{2}{|c|}{ Memory } & \multicolumn{2}{|c|}{$\begin{array}{c}\text { Fluency } \\
\text { phonological }\end{array}$} \\
\hline & Rho & $\mathbf{p}$ & Rho & $\mathbf{p}$ & Rho & $\mathbf{p}$ & Rho & $\mathbf{p}$ & Rho & $\mathbf{P}$ & Rho & $\mathbf{p}$ \\
\hline $\begin{array}{l}\text { Fractional anisotropy } \\
\text { Right cerebral peduncle } \\
\text { Left cerebral peduncle } \\
\text { Right external capsule } \\
\text { Left cingulum gyrus }\end{array}$ & $\begin{array}{l}0.31 \\
0.72 \\
0.34 \\
0.60\end{array}$ & $\begin{array}{l}0.44 \\
0.04 \\
0.40 \\
0.11\end{array}$ & $\begin{array}{c}0.03 \\
0.07 \\
-0.27 \\
-0.10\end{array}$ & $\begin{array}{l}0.93 \\
0.86 \\
0.50 \\
0.79\end{array}$ & $\begin{array}{l}-0.10 \\
-0.07 \\
-0.35 \\
-0.12\end{array}$ & $\begin{array}{l}0.81 \\
0.86 \\
0.39 \\
0.76\end{array}$ & $\begin{array}{c}0.11 \\
-0.27 \\
0.07 \\
0.73\end{array}$ & $\begin{array}{l}0.78 \\
0.51 \\
0.85 \\
0.04\end{array}$ & $\begin{array}{c}-0.32 \\
-0.70 \\
-0.18 \\
0.25\end{array}$ & $\begin{array}{l}0.43 \\
0.05 \\
0.67 \\
0.54\end{array}$ & $\begin{array}{c}-0.36 \\
-0.72 \\
-0.44 \\
0.32\end{array}$ & $\begin{array}{l}0.37 \\
0.04 \\
0.26 \\
0.43\end{array}$ \\
\hline $\begin{array}{l}\text { Mean diffusivity } \\
\text { Right thalamic posterior radiation } \\
\text { Left external capsule }\end{array}$ & $\begin{array}{l}-0.16 \\
-0.46\end{array}$ & $\begin{array}{l}0.69 \\
0.25\end{array}$ & $\begin{array}{l}-0.12 \\
-0.20\end{array}$ & $\begin{array}{l}0.77 \\
0.62\end{array}$ & $\begin{array}{l}-0.10 \\
-0.12\end{array}$ & $\begin{array}{l}0.81 \\
0.76\end{array}$ & $\begin{array}{l}-0.39 \\
-0.54\end{array}$ & $\begin{array}{l}0.33 \\
0.16\end{array}$ & $\begin{array}{c}0.24 \\
-0.46\end{array}$ & $\begin{array}{l}0.56 \\
0.24\end{array}$ & $\begin{array}{c}0.21 \\
-0.27\end{array}$ & $\begin{array}{l}0.60 \\
0.50\end{array}$ \\
\hline
\end{tabular}

without ventricles 904.25 versus $837.76(p<0.01)$, brain segmentation volume without ventricles from surf 903.46 versus $837.16(p<0.01)$, right hemisphere cerebral white matter volume 184.52 versus 168.32 $(p<0.03)$, subcortical gray matter volume 46.06 versus $42.10(p<0.05)$, total volume of gray matter 514.03 versus 479.94 ( $p<0.01$ ), total intracranial volume 1471.92 versus $1324.38(p<0.05)$, left thalamus 5.86 versus $5.37(p<0.02)$, left caudate 3.5 versus 2.71 $(p<0.02)$, fourth ventricle 2.14 versus $1.54(p<0.05)$, right caudate 4.01 versus $2.90(p<0.02)$, and right choroid plexus 0.92 versus $0.71(p<0.03)$ (Table 4).

\section{Discussion}

Our study compared patients with $A D$ and MixD through a standardized acquisition protocol to investigate the parameters of FA and MD in terms of changes in the anisotropy or magnitude of water diffusion throughout the brain, demonstrating that patients with $A D$ have alterations in specific tracts, such as right and left cerebral peduncle, right and left external capsule, left cingulate gyrus, and right thalamic posterior radiation compared to patients with MixD.

Palesi et al. demonstrated in a tractography study in patients with $D V a, A D$, and a control group that patients with $A D$ had a greater affection in the parahippocampal tracts and in the knee of the corpus callosum, while patients with DVa showed a greater affection of white matter in thalamic radiation ${ }^{28}$. Reginold et al., in a study which objective was to evaluate the affection of white matter in patients with $A D$, demonstrated a greater commitment in the superficial white matter of the temporal lobe than in the members of a control group ${ }^{25}$.

Tu et al. compared DTI parameters in members of a control group, patients with $A D$ and subcortical ischemic vascular disease (SIVD; also called lacunar infarction), reporting a global decrease in FA in patients with SIVD; while in patients with $A D$, the alterations were in the left superior longitudinal bundle, knee and splenium of the corpus callosum, anterior thalamic radiation, uncinate bundle, and left cingulate gyrus, suggesting that DTI is effective in distinguishing patients with early stage of $A D$ versus SIVD ${ }^{29}$. Another 
Table 4. Volumetry in regions of interest in Alzheimer's disease and mixed dementia

\begin{tabular}{|c|c|c|c|c|}
\hline & $\begin{array}{l}\text { Total patients } \\
(n=16)\end{array}$ & $\begin{array}{l}\text { Alzheimer's disease } \\
\qquad(\mathrm{n}=\mathbf{8})\end{array}$ & $\begin{array}{l}\text { Mixed dementia } \\
\qquad(\mathrm{n}=\mathbf{8})\end{array}$ & p \\
\hline Brain segmentation Volume, $\mathrm{cm}^{3}$ & $921,44 \pm 76.83$ & $964.92 \pm 69.93$ & $877.95 \pm 58.61$ & 0.02 \\
\hline Brain segmentation volume without ventricles, $\mathrm{cm}^{3}$ & $871.00 \pm 58.93$ & $904.25 \pm 47.37$ & $837.76 \pm 51.68$ & 0.01 \\
\hline Brain segmentation volume without ventricles from surf, $\mathrm{cm}^{3}$ & $870.31 \pm 58.87$ & $903.46 \pm 47.38$ & $837.16 \pm 51.67$ & 0.01 \\
\hline Right hemisphere cerebral white matter volume, $\mathrm{cm}^{3}$ & $176.42 \pm 16.08$ & $184.52 \pm 14.60$ & $168.32 \pm 13.83$ & 0.03 \\
\hline Subcortical gray matter volume, $\mathrm{cm}^{3}$ & $44.08 \pm 3.81$ & $46.06 \pm 3.36$ & $42.10 \pm 3.30$ & 0.05 \\
\hline Total volume of gray matter, $\mathrm{cm}^{3}$ & $496.99 \pm 34.42$ & $514.03 \pm(24.91)$ & $479.94 \pm 35.42$ & 0.01 \\
\hline Total intracranial volume, $\mathrm{cm}^{3}$ & $1398.15 \pm 150.91$ & $1471.92 \pm 118.28$ & $1324.38 \pm 149.58$ & 0.05 \\
\hline Left thalamus, $\mathrm{cm}^{3}$ & $5.62 \pm 0.43$ & $5.86 \pm 0.39$ & $5.37 \pm 0.33$ & 0.02 \\
\hline Left caudate, $\mathrm{cm}^{3}$ & $3.11 \pm 0.69$ & $3.5 \pm 0.64$ & $2.71 \pm 0.47$ & 0.02 \\
\hline Fourth ventricle, $\mathrm{cm}^{3}$ & $1.84 \pm 0.53$ & $2.14 \pm 0.45$ & $1.54 \pm 0.45$ & 0.05 \\
\hline Right caudate, $\mathrm{cm}^{3}$ & $3.45 \pm 0.10$ & $4.01 \pm 0,24$ & $2.90 \pm 0.51$ & 0.02 \\
\hline Right choroid plexus, $\mathrm{cm}^{3}$ & $0.82 \pm 0.25$ & $0.92 \pm 0.27$ & $0.71 \pm 0.21$ & 0.03 \\
\hline
\end{tabular}

study by Lee et al., which compared control group members, patients with $\mathrm{MCl}$ and $\mathrm{AD}$, showed that the latter group had a greater decrease in FA, as well as lower integrity of white matter associated with a lower hippocampal volume, indicating that the pathology in white matter follows the same degree of stages and progression of neurodegeneration ${ }^{30}$.

Another finding in our study was the combined affection of tracts corresponding to neurodegeneration (turn of the left cingulum, cerebral peduncles) $)^{31}$, as well as areas related to the vascular component (thalamic radiation ${ }^{25}$, a combination only observed, in our study, in patients with MixD. These alterations are the result of changes in axonal density and myelination since the homogeneity in the orientation of the axons affects the degree of FA and MD in DTI, so a decrease in FA and an increase in MD reflect a decrease in the integrity of the brain tissue ${ }^{1,6,32}$. An important aspect to consider is the fact that the vascular load in white matter in patients with MixD could be a potential confounder, similar to other DTI studies in neurodegenerative diseases.

In relation to the results of volumetry, a lower volume was observed in twelve ROIs in patients with MixD. Some studies have established a correlation between DTI parameters and volumetric parameters, indicating that these are measures of the same pathological process, that is, neurodegeneration ${ }^{33}$. The relative change in the parameters that reflect cortical integrity and diffusion is likely to vary not only depending on the methods used but also on the particular morphological process, the anatomical region being studied, and possibly the underlying molecular pathology of the disease in particular ${ }^{27}$. One aspect of techniques for detecting changes in white matter tracts is that they are more sensitive than measurements of cortical thickness or integrity; this is justified because the loss of cortical volume in neurodegenerative disease probably represents the loss of individual neurons (soma) and neuropil (corresponding dendrites, axons) $)^{4,28}$.

Regarding the cognitive domains and the affected tracts, we found an association between FA and orientation (left cerebral peduncle) in patients with a diagnosis of $A D$; this anatomical structure is considered a vulnerable area from the vascular point of view ${ }^{34}$, in addition, it is related to changes in $A D^{29}$. An association was also observed between FA and the orientation domains (left cingulate), as well as in-memory and phonological fluency (left cerebral peduncle) in patients with MixD; these findings show that there is a disconnection of areas related to cortical memory (left cingulate) since this structure is a fundamental part of the limbic system and one of the main fascicles of white matter that connects areas of cortical association ${ }^{35}$.

Our results suggest that DTI allows the identification of alterations in specific tracts (cerebral peduncle and cingulate) in patients with AD compared to patients with MixD. This shows that MixD shares a spectrum of neurodegeneration (due to the affection of 
classic tracts affected in AD) but with alterations (decrease) in FA.

The limitations of our study are based on the cross-sectional design, which does not allow the interpretation of the causal mechanisms underlying the associations of MRI and cognitive measurements. Another important aspect is the size of the sample since this could limit the ability to detect more differences in DTI between patients with $A D$ and MixD. Although there are various computer programs available that allow tractography to be carried out, some even free to use for research purposes, the results obtained with one or the other may vary, either due to the algorithm they use or due to their processing, since the reproducibility depends to some degree on the user's interaction with the computer program. In multicenter studies, there are also variations due to different MRI equipment and the protocol used to acquire DTI images; these can influence the quantification of the results using tractography, as mentioned by Fischer et al. and Heiervang et al., who found differences in the quantification of the cerebral fascicles applying different diffusion gradients.

Our study has several strengths: it is the first study to describe tractography characteristics and their association with cognitive performance in elderly adults with AD and MixD. Our findings could lead to future studies in which MixD can be characterized better and the role of tractography and volumetry can be understood more clearly.

Our results support existing findings reported in the literature and, most importantly, provide a complete interpretation of microstructural alterations in the white matter through the spectrum of dementia type $A D$ and MixD using the tractography technique with parametric analysis, and it could provide a useful standard for the early diagnosis of $A D$ and MixD in the future.

\section{Conclusion}

Tractography is an effective method to distinguish alterations in brain tracts through the measurement of the anisotropy fraction since these alterations show that MixD shares a spectrum of neurodegeneration and vascular affection.

\section{Funding}

This research received no specific grant from any funding agency in public, commercial, or not-for-profit sectors.

\section{Conflicts of interest}

None.

\section{Ethical disclosures}

Protection of human and animal subjects. The authors declare that no experiments were performed on humans or animals for this study.

Confidentiality of the data. The authors declare that they have followed the protocols of their work center on the publication of patient data.

Right to privacy and informed consent. The authors declare that no patient data appear in this article.

\section{References}

1. Larroza A, Moratal D, D’ocón V, Arana E. Tractografía del fascículo uncinado y el fascículo uncinado posterior en pacientes con deterioro cognitivo leve y enfermedad de Alzheimer. Neurology. 2014;29:11-20.

2. Vilanova A, Zhang S, Kindlmann G, Laidlaw D. An introduction to visualization of diffusion tensor imaging and its applications. Vis Appl. 2006;15:121-53.

3. Lazar M. Mapping brain anatomical connectivity using white matter tractography. NMR Biomed. 2010;23:821-35.

4. Reginold W, Luedke A, Itorralba J, Fernandez J, Islam O, García A, et al. Altered superficial white matter on tractography MRI in Alzheimer's disease. Dement Geriatr Cogn Disord Extra. 2016;6:233-41.

5. Kvickström P, Eriksson B, Van Westen D, Lätt J, Elfgren C, Nilsson C Selective frontal neurodegeneration of the inferior fronto-occipital fasciculus in progressive supranuclear palsy (PSP) demonstrated by diffusion tensor tractography. BMC Neurol. 2011;11:1-8.

6. Acosta J, Guy B, Pengas G, Peter J. Absolute diffusivities define the landscape of white matter degeneration in Alzheimer's disease. Brain. 2010;133:529-39.

7. Le Bihan D. Looking into the functional architecture of the brain with diffusion MRI. Nat Rev Neurosci. 2003;4:469-80.

8. Brickell KL, Steinbart EJ, Rumbaugh M, Haydeh P, Schellenberg G, Van Deerlin V, et al. Early-onset Alzheimer disease in families with late-onse Alzheimer disease: a potential important subtype of familial Alzheimer disease. Arch Neurol. 2006;63:1307-11.

9. Moreno C, Mimenza J, Aguilar S, Alvarado P, Gutiérrez L, Juárez S, et al. Factores asociados a la demencia mixta en comparación con la demencia tipo Alzheimer en adultos mayores mexicanos. Neurology. 2017;32:309-15.

10. Korczyn A. Mixed dementia: the most common cause of dementia? Can J Diagn. 2010;9:25-44.

11. Skrobot O, O'Brien J, Black S, Chend C, DeCarlie C, Erkinjunttif T, et al. Progress toward standardized diagnosis of vascular cognitive impairment: guidelines from the vascular impairment of cognition classification consensus study. Alzheimers Dement. 2018;14:280-92

12. Zekry D, Hauw JJ, Gold G. Mixed dementia: epidemiology, diagnosis, and treatment. J Am Geriatr Soc. 2002;50:1431-8.

13. Katz S, Akpom CA. Index of ADL. Med Care. 1976;14:116-8.

14. Lawton MP, Brody EM. Assessment of older people: self-mantaining and instrumental activities of daily living. Gerontologist. 1969;9:179-86.

15. Sheikh JI, Yesavage JA. Geriatric depression scale (GDS): recent evidence and development of a shorter version. Clin Gerontol. 1986;5:165-72.

16. Ostrosky-Solis F, Ardila A, Rosselli M. NEUROPSI: a brief neuropsychological test battery in Spanish with norms by age and educational level. J Int Neuropsychol Soc. 1999;5:413-33.

17. Folstein M, Folstein S, McHugh P. Mini-mental state. A practical method for grading the cognitive state of patients for the clinician. J Psychiatr Res 1975;12:189-98.

18. Lima E. Role of neuropsychological assessment in the differential diagnosis of Alzheimer's disease and vascular dementia. Dement Neuropsychol. 2009;3:214-21.

19. Aguilar S, Mimenza A, Samudio M, Hernández F, Gutiérrez L, Ramírez F, et al. Validation of the clock drawing test scoring method in older adults with neurocognitive disorder. Salud Ment. 2018;41:179-86.

20. Rodríguez A. FAB: aplicación preliminar española de la batería neuropsicológica de evaluación de funciones frontales a 11 grupos de pacientes. Rev Neurol. 2003;36:605-8. 
Rev Mex Neuroci. 2021;22(2)

21. Morris JC. The clinical dementia rating (CDR): current version and scoring rules. Neurology. 1993;43:2412-4.

22. Guía de Consulta de los Criterios Diagnósticos del DSM-5®. Spanish Edition of the Desk Reference to the Diagnostic Criteria From DSM-5®. Philadelphia, PA: American Psychiatric Publisher; 2014. p. 314-5.

23. McKhann G, Drachman D, Folstein M, Katzman R, Price D, Stadian EM. Clinical diagnosis of Alzheimer's disease; report of the NINCDS-ADRDA work group under the auspices of department of health and human services task force on Alzheimer's disease. Neurology. 1984;34:939-44.

24. Reginold W, Itorralba J, Luedke A, Fernandez-Ruiz J, Reginold J, Islam $\mathrm{O}$, et al. Tractography at $3 \mathrm{~T}$ MRI of corpus callosum tracts crossing white matter hyperintensities. Am J Neuroradiol. 2016;37:1617-22.

25. Reginol W, Luedke A, Tam A, Itorralba J, Fernandez J, Reginold J, et al. Cognitive function and 3-tesla magnetic resonance imaging tractography of white matter hyperintensities in elderly persons. Dement Geriatr Cogn Disord Extra. 2015;5:387-94

26. Sedrak M, Gorgulho A, Bari A, Behnke E, Frew A, Gevorkyan I, et al Diffusion tensor imaging (DTI) and colored fractional anisotropy (FA) mapping of the subthalamic nucleus (STN) and the globus pallidus interna (GPi). Acta Neurochir (Wien). 2010;152:2079-84.

27. Santillo A, Martensson J, Lindberg O, Nilsson M, Manzouri A Landqvist $\mathrm{M}$, et al. Diffusion tensor tractography versus volumetric imaging in the diagnosis of behavioral variant frontotemporal dementia. PLoS One. 2013;8:1-9.
28. Palesi F, De Rinaldis A, Vitali P, Castellazzi G, Casiraghi L, Germani G, et al. Specific patterns of white matter alterations help distinguishing Alzheimer's and vascular dementia. Front Neurosci. 2018;274:1-14.

29. Chien $\mathrm{M}$, Chung-Ping $\mathrm{L}$, Ching-Feng $\mathrm{H}$, Yen-Hsuan $\mathrm{H}$, Wen-Hui $\mathrm{H}$ Deng J, et al. Effectiveness of diffusion tensor imaging in differentiating early-stage subcortical ischemic vascular disease, Alzheimer's disease and normal ageing. PLoS One. 2017;12:e0175143.

30. Seung L, Coutua J, Wilkensa P, Yendikia A, Rosasa D, Salata D, et al. Tract-based analysis of white matter degeneration in Alzheimer's disease. Neuroscience. 2015;20:79-89.

31. Canu E, McLaren D, Fitzgerald M, Bendlin B, Zoccatelli G, Alessandrini F, et al. Mapping the structural brain changes in Alzheimer's disease: the independent contribution of two imaging modalities. J Alzheimers Dis 2011;26:263-74.

32. Alves G, Oertel V, Knöchel C, Carvalho A, Pantel J, Engelhardt E, et al. Integrating retrogenesis theory to Alzheimer's disease pathology: insight from DTI-TBSS investigation of the white matter microstructural integrity. Biomed Res Int. 2015;2015:291658.

33. Avants B, Cook P. Ungar L, Gee J, Grossman M. Dementia induces correlated reductions in white matter integrity and cortical thickness: a multivariate neuroimaging study with sparse canonical correlation analysis. Neuroimage 2010;50:1004-16.

34. Narayana S, Glikstein R, Hanagandil P, Chakraborty S. Role of neuroimaging in multidisciplinary approach towards non-Alzheimer's dementia. Insights Imaging 2015;6:531-44

35. Bozzali M, Giulietti G, Basile B, Serra L, Spanò B, Perri R, et al. Damage to the cingulum contributes to Alzheimer's disease pathophysiology by deafferentation mechanism. Hum Brain Mapp. 2012;33:1295-308. 